\title{
Influence of Drip Irrigation Levels and Soilless Media on the Growth, Productivity and Economics of Greenhouse Grown Tomato
}

\author{
R. Parameshwarareddy ${ }^{1 *}$, S.S. Angadi ${ }^{1}$, R.H. Patil ${ }^{1}$ and M.S. Biradar ${ }^{2}$ \\ ${ }^{1}$ Department of Agronomy, University of Agricultural Sciences, Dharwad, Karnataka, India \\ ${ }^{2}$ Department of Horticulture, University of Agricultural Sciences, Dharwad, Karnataka, India \\ *Corresponding author
}

\section{A B S T R A C T}

\begin{tabular}{|l|}
\hline K e y w o r d s \\
Drip irrigation, \\
Greenhouse grown \\
tomato
\end{tabular}

The study was carried out to determine the suitable irrigation levels and soilless media for fresh market of tomato under greenhouse condition, at Hi-tech Horticulture unit, Saidapur farm, MARS, University of Agricultural Sciences, Dharwad. The experiment was laid out in CRD with two factors viz., as factor I- drip irrigation at once a day $\left(\mathrm{I}_{1}: 50 \% \mathrm{PE}, \mathrm{I}_{2}: 75\right.$ $\% \mathrm{PE} \mathrm{I}_{3}: 100 \% \mathrm{PE}$ ) and factor-II includes $\mathrm{M}_{1}$ : Cocopeat, $\mathrm{M}_{2}$ :Perlite, $\mathrm{M}_{3}$ :Vermiculite, $\mathrm{M}_{4}$ Cocopeat + Perlite (50:50) - (1:1), $\mathbf{M}_{5}$ : Cocopeat + Vermiculite (50:50) - (1:1), $\mathbf{M}_{6}$ : Cocopeat + Perlite + Vermiculite (50:25:25)- (2:1:1), $\mathbf{M}_{7}$ : Sole soil (Grown in earthen pot) $\mathrm{M}_{8}$ : Sole soil (Grown under normal condition). The highest fruit yield $\left(126.7 \mathrm{t} \mathrm{ha}^{-1}\right.$ ) and fruit weight $(46.77 \mathrm{~g})$ were obtained from drip irrigation at 100 per cent pan evaporation with cocopeat + perlite + vermiculite $(50: 25: 25)$. Similarly, $\mathrm{I}_{3} \mathbf{M}_{6}$ combination noticed higher gross returns (Rs. $1520334 \mathrm{ha}^{-1}$ ) and net returns (Rs.797877 ha ${ }^{-1}$ ) over other treatment combinations

\section{Introduction}

Soil is the most available growing medium for plants. It provides nutrients, air and water, etc. for plant growth. Soil has serious limitations for plant growth, due to the presence of disease causing organisms and nematodes, unsuitable soil reaction, unfavourable soil compaction, poor drainage, degradation due to erosion etc., Conventional agriculture is difficult to practice as it involves large area, lot of labour, climate change and large volume of water for cultivation. In urban areas, quality soil is less available for crop growing or in some areas, there is scarcity of fertile cultivable arable lands due to their unfavorable geographical conditions. Under such situations, soilless culture can be introduced successfully (Butler and Oebker, 2006). Besides, in areas where soil is not present soilless culture can be introduced. The crops suitable for practicing the soilless culture includes cereals (Rice and Maize), vegetables (tomato, chilli, brinjal etc.), leafy vegetables (lettuce), flower crops (rose, marigold etc.) and fodder crops (sorghum, barley etc.) (Sardare and Admane, 2013).

Today our aim is to increase the agricultural production per unit volume of water, per unit cropped land in a unit time. The slogan of the day "more crop per drop" more appropriately 
emphasizes the same. The scientific management of irrigation water provides the insurance against weather induced fluctuation in total production. This is the only way in which we can make our agriculture competitive and profitable. Hence, a pot experiment was conducted to evaluate the performance of tomato with irrigation levels and soilless media under protected condition.

\section{Materials and Methods}

The experiment was conducted at the Hi-tech Horticulture unit at Saidapur Farm, MARS, UAS, Dharwad which is situated in the Northern Transition Zone (Zone 8) in Karnataka state. The location corresponds to $15^{\circ} 26^{\prime} \mathrm{N}$ latitude and $75^{\circ} 07^{\prime}$ East longitude with an altitude of $730 \mathrm{~m}$ above the mean sea level (MSL). Tomato was raised in a naturally ventilated polyhouse. It was made up of galvanized iron pipes and covered with 200 micron UV stabilized polythene sheet and the two sides were covered with insect net for natural ventilation to protect against insect pests. Besides, a rollable flap polyethylene sheet has also been provided outside the insect net to regulate the requirements of temperature and humidity depending on the season and weather conditions. The shade net with 50 per cent shade was laid out at above the headspace inside the greenhouse to manage the light intensity and temperature during summer. The experiment was laid out using two factor CRD design with tomato hybrid STH-801 as the test crop. The growing system designed for this particular study was earthen pots of size 8.51 capacity. Three different inert growing media combinations were used for the experiment and soil was used as check. The treatment (twenty four) comprised the combination of drip irrigation $\left(\mathrm{I}_{1}: 50\right.$ per cent of pan evaporation $\mathrm{I}_{2}: 75$ per cent of pan evaporation $\mathrm{I}_{3}: 100$ per cent of pan evaporation) as factor-I and medias $\left(\mathrm{M}_{1}\right.$ : Cocopeat, $\mathrm{M}_{2}$ :Perlite, $\mathrm{M}_{3}$ :Vermiculite, $\mathrm{M}_{4}$ Cocopeat + Perlite
$(50: 50)-(1: 1), M_{5}$ : Cocopeat + Vermiculite $(50: 50)-(1: 1), \mathrm{M}_{6}$ : Cocopeat + Perlite + Vermiculite (50:25:25)- (2:1:1), $\mathrm{M}_{7}$ : Sole soil (Grown in earthen pot) $\mathrm{M}_{8}$ : Sole soil (Grown under normal condition) as factor -II. The daily water requirement for drip irrigation was computed using the data from Class-A open pan evaporimeter.

\section{Results and Discussion}

\section{Response of tomato to drip irrigation levels}

The yield of tomato is the cumulative expression of yield attributing characters such as fruit yield plant ${ }^{-1}$, fruit weight, per cent fruit set, number of fruits cluster ${ }^{-1}$, number flowers cluster $^{-1}$ and number flower clusters plant $^{-1}$ which differed significantly with drip irrigation levels (Table 1). Number of fruits plant $^{-1}$ is an important yield attributing character which determines total yield. Higher number of fruits plant ${ }^{-1}$ (55.0) achieved with drip irrigation at 100 per cent PE. Maintenance of optimum moisture in the root zone helps to utilize nutrients properly. The essential nutrients supplied through drip irrigation constituted an increase in number of fruits plant ${ }^{-1}$. The fertigation was scheduled with the balanced amount of macronutrients and micronutrients. The macronutrients like nitrogen, phosphorus and potassium played a vital role in accelerating the process of cell division and elongation and hence resulted in more number of fruits cluster ${ }^{-1}$. The fruit yield $\left(105.9 \mathrm{t} \mathrm{ha}^{-1}\right)$ is nothing but the resultant of higher number of fruits plant $^{-1}$ and fruit weight which were significantly higher at drip irrigation with 100 per cent PE. Similarly, number of flowers cluster ${ }^{-1}$ (6.7), per cent fruit set $(91.7 \%)$ and number of flower clusters plant $^{-1}$ (9.0) were also differed significantly with 100 per cent PE, due to continuous availability of moisture throughout the crop period. These results are in conformity with the findings of Sezen et al., (2010). On the 
contrary, drip irrigation at 50 per cent PE recorded significantly lower yield $(64.71 \mathrm{t}$ $\left.\mathrm{ha}^{-1}\right)$. Yield reduction was due to significant reduction in yield attributing characters, such as, fruit weight $(43.37 \mathrm{~g})$, per cent fruit set $(88.3 \%)$, and number of flower clusters plant ${ }^{-1}$ (7.4). The significant reduction in yield attributes with drip irrigation at 50 per cent $\mathrm{PE}$ might be due to reduction in availability of moisture in the root zone as compared to other irrigation levels. This concept not only involves the final yield and its components, but also probes into physiological events that have occurred early in growth stage causing variation in growth and yield of crop. It is well established fact that the infrastructure of the plant is decided by growth parameters such as plant height and number of branches.

Different irrigation levels had a significant influence on all the growth parameters. Whereas, days to fifty per cent flowering (47.00 days) was higher at drip irrigation with 100 per cent PE as compared to other levels.

Plant height is one of the important growth parameters had an indirect effect on yield of tomato. As the plant height increases, there may be more light interception and dry matter accumulation which were reflected in higher yield. In the present investigation, drip irrigation with 100 per cent PE recorded significantly higher plant height (198.7, 135.9 and $73.9 \mathrm{~cm}$ at 30, 60 DAT and harvest respectively) and number of branches (8.1,21.8 and 29.9 at 30, 60 DAT and harvest respectively) over other levels. Similar findings were obtained by Dunage et al., (2009). The increment in plant height was due to higher availability of nutrients which accelerated the cell division and elongation. Whereas, drip irrigation at 50 per PE recorded significantly lower plant height $(149.6 \mathrm{~cm})$. The reduction in plant height might be due to poor nutrition as well as less water supply to plants.
Response of tomato to various Soilless media

In the present study different combinations of substrates like cocopeat, perlite, vermiculite, cocopeat + perlite at 50:50, cocopeat + vermiculite at 50:50, cocopeat + perlite + vermiculite (50:25:25), sole soil (Grown in earthen pot) and normal soil grown conditions.

The substrates had significant influence on fruit yield of tomato. Mixed substrates of cocopeat + perlite + vermiculite at 50:25:25 recorded (Table 2) significantly higher yield $\left(100.90 \mathrm{t} \mathrm{ha}^{-1}\right)$ over other substrates. Similar findings were obtained by $\mathrm{He}$ et al., (2003), Joseph and Muthuchamy (2014). The increase in yield under cocopeat + perlite + vermiculite at 50:25:25 might be due to an increase in yield attributes like fruit weight $(43.3 \mathrm{~g})$ and per cent fruit set $(89.3 \%)$. However, it was on par with cocopeat + perlite at 50:50 and cocopeat + vermiculite at 50:50. The increase in yield attributes was probably associated with the conservation of moisture and improved micro-climate in soilless conditions.

Owing to quicker root growth and development of tomato in cocopeat resulted in better yield. The fruit weight, number of flowers cluster ${ }^{-1}$, number fruits cluster $^{-1}$ number of flower clusters plant ${ }^{-1}$ and days to fifty per cent flowering were on par with soilless media as compared to others.

Plant height $(183.9 \mathrm{~cm})$ recorded under cocopeat + perlite + vermiculite at 50:25:25 was significantly higher over other substrates. Similar findings were reported by Haddad (2007). However, it was on par with cocopeat + perlite at 50:50 $(181.4 \mathrm{~cm})$, cocopeat + vermiculite at 50:50 $(180.6 \mathrm{~cm})$. This could be due to no chance of soil-borne insect pest, disease attack or weed infestation which helped in efficient nutrient regulation and thus leading to increase in yield of tomato. 
Table.1 Growth and yield attributes of tomato as influenced by irrigation levels and different substrates

\begin{tabular}{|c|c|c|c|c|c|c|c|c|c|c|}
\hline \multirow[t]{2}{*}{ Treatment } & \multicolumn{3}{|c|}{ Plant height } & \multicolumn{3}{|c|}{ No. of. branches } & \multirow{2}{*}{$\begin{array}{c}\text { Days to } \\
\text { fifty } \\
\text { flowering }\end{array}$} & \multirow{2}{*}{$\begin{array}{l}\text { No. of. Flower } \\
\text { clusters plant }^{-1}\end{array}$} & \multirow{2}{*}{$\begin{array}{l}\text { No. of. } \\
\text { Flowers } \\
\text { cluster }^{-1}\end{array}$} & \multirow{2}{*}{$\begin{array}{l}\text { No. of. } \\
\text { Fruits } \\
\text { cluster }\end{array}$} \\
\hline & 30 DAT & $\begin{array}{c}60 \\
\text { DAT }\end{array}$ & $\begin{array}{c}\text { At } \\
\text { harvest }\end{array}$ & $\begin{array}{c}30 \\
\text { DAT }\end{array}$ & 60 DAT & At harvest & & & & \\
\hline \multicolumn{11}{|l|}{ Irrigation (I) } \\
\hline $\mathrm{I}_{1}$ & 56.8 & 100.4 & 149.6 & 5.7 & 14.8 & 25.7 & 41.7 & 7.5 & 5.5 & 4.8 \\
\hline$I_{2}$ & 64.5 & 124.2 & 171.9 & 6.9 & 18.0 & 27.8 & 45.4 & 8.2 & 6.1 & 5.5 \\
\hline $\mathbf{I}_{3}$ & 73.9 & 135.9 & 198.7 & 8.1 & 21.8 & 29.9 & 47.0 & 9.0 & 6.7 & 6.1 \\
\hline S.Em \pm & 0.65 & 1.06 & 1.01 & 0.14 & 0.22 & 0.32 & 0.38 & 0.16 & 0.14 & 0.12 \\
\hline C.D. at $1 \%$ & 2.45 & 4.01 & 3.84 & 0.55 & 0.85 & 1.20 & 1.45 & 0.61 & 0.52 & 0.44 \\
\hline \multicolumn{11}{|l|}{ Media (M) } \\
\hline $\mathrm{M}_{1}$ & 66.6 & 119.8 & 175.1 & 6.8 & 18.0 & 27.7 & 45.0 & 8.3 & 6.2 & 5.6 \\
\hline $\mathrm{M}_{2}$ & 67.6 & 121.2 & 176.4 & 7.0 & 18.6 & 28.0 & 44.6 & 8.3 & 6.2 & 5.6 \\
\hline$\overline{\mathbf{M}_{3}}$ & 67.7 & 120.7 & 176.2 & 7.0 & 18.4 & 28.2 & 44.6 & 8.3 & 6.4 & 5.8 \\
\hline $\mathbf{M}_{4}$ & 67.1 & 124.4 & 181.4 & 7.3 & 19.0 & 29.1 & 45.7 & 8.8 & 6.2 & 5.7 \\
\hline $\mathbf{M}_{5}$ & 69.3 & 126.2 & 180.6 & 7.4 & 19.3 & 29.4 & 45.3 & 8.6 & 6.3 & 5.8 \\
\hline $\mathrm{M}_{6}$ & 70.6 & 128.4 & 183.9 & 7.9 & 20.4 & 29.7 & 46.1 & 8.9 & 6.6 & 5.9 \\
\hline $\mathbf{M}_{7}$ & 53.1 & 106.7 & 151.4 & 5.6 & 15.3 & 24.0 & 42.4 & 6.8 & 5.1 & 4.6 \\
\hline $\mathbf{M}_{8}$ & 58.3 & 114.1 & 162.1 & 6.1 & 16.6 & 26.1 & 44.0 & 7.7 & 5.4 & 4.9 \\
\hline S.Em \pm & 1.06 & 1.73 & 1.65 & 0.24 & 0.36 & 0.52 & 0.62 & 0.26 & 0.22 & 0.19 \\
\hline C.D. at $1 \%$ & 4.01 & 6.56 & 6.27 & 0.89 & 1.38 & 1.97 & 2.36 & 0.99 & 0.84 & 0.72 \\
\hline \multicolumn{11}{|l|}{ Interaction } \\
\hline $\mathbf{I}_{1} \mathbf{M}_{1}$ & 56.7 & 100.7 & 151.3 & 5.7 & 15.0 & 26.0 & 42.7 & 7.3 & 5.67 & 4.98 \\
\hline $\mathbf{I}_{1} \mathbf{M}_{2}$ & 58.7 & 100.3 & 153.0 & 6.0 & 15.0 & 26.0 & 41.7 & 7.3 & 5.67 & 5.04 \\
\hline $\mathbf{I}_{1} \mathbf{M}_{3}$ & 59.7 & 101.7 & 152.3 & 5.7 & 14.7 & 26.3 & 41.7 & 7.3 & 6.00 & 5.37 \\
\hline $\mathbf{I}_{1} \mathbf{M}_{4}$ & 58.7 & 104.0 & 155.0 & 6.0 & 15.7 & 27.0 & 42.3 & 8.3 & 5.33 & 4.69 \\
\hline $\mathbf{I}_{1} \mathbf{M}_{5}$ & 61.0 & 105.0 & 154.3 & 6.3 & 15.3 & 27.3 & 41.3 & 7.7 & 5.67 & 5.07 \\
\hline $\mathbf{I}_{1} \mathbf{M}_{6}$ & 61.3 & 107.3 & 157.3 & 6.7 & 16.3 & 27.7 & 42.7 & 8.3 & 5.67 & 4.94 \\
\hline $\mathbf{I}_{1} \mathbf{M}_{7}$ & 46.3 & 88.0 & 132.0 & 4.3 & 13.0 & 21.3 & 39.7 & 6.3 & 5.00 & 4.41 \\
\hline $\mathbf{I}_{1} \mathbf{M}_{8}$ & 51.7 & 96.3 & 141.6 & 4.7 & 13.7 & 23.7 & 41.7 & 7.0 & 4.67 & 4.05 \\
\hline $\mathbf{I}_{2} \mathbf{M}_{1}$ & 66.0 & 124.0 & 175.0 & 6.3 & 17.0 & 28.0 & 45.3 & 8.3 & 6.33 & 5.80 \\
\hline $\mathrm{I}_{2} \mathrm{M}_{2}$ & 66.7 & 125.3 & 176.3 & 7.3 & 18.0 & 28.3 & 45.3 & 8.3 & 6.33 & 5.81 \\
\hline $\mathrm{I}_{2} \mathrm{M}_{3}$ & 67.3 & 124.7 & 175.6 & 7.0 & 18.0 & 28.7 & 45.0 & 8.3 & 6.67 & 6.00 \\
\hline $\mathbf{I}_{2} \mathbf{M}_{4}$ & 67.0 & 129.7 & 178.3 & 7.3 & 18.3 & 29.3 & 46.7 & 8.7 & 6.33 & 5.86 \\
\hline $\mathbf{I}_{2} \mathbf{M}_{5}$ & 68.0 & 131.3 & 177.7 & 7.3 & 19.0 & 29.7 & 46.3 & 8.7 & 6.33 & 5.78 \\
\hline $\mathrm{I}_{2} \mathrm{M}_{6}$ & 70.3 & 132.0 & 179.7 & 7.7 & 20.3 & 29.3 & 47.0 & 8.7 & 6.67 & 6.07 \\
\hline $\mathbf{I}_{2} \mathbf{M}_{7}$ & 53.3 & 112.0 & 153.7 & 5.7 & 16.0 & 23.7 & 42.7 & 6.7 & 4.67 & 4.26 \\
\hline $\mathbf{I}_{2} \mathbf{M}_{8}$ & 57.3 & 114.7 & 158.7 & 6.3 & 17.0 & 25.0 & 44.7 & 7.7 & 5.33 & 4.79 \\
\hline $\mathbf{I}_{3} \mathbf{M}_{1}$ & 77.2 & 134.7 & 199.0 & 8.3 & 22.0 & 29.0 & 47.0 & 9.3 & 6.67 & 6.05 \\
\hline $\mathbf{I}_{\mathbf{3}} \mathbf{M}_{\mathbf{2}}$ & 77.3 & 137.3 & 200.0 & 7.7 & 22.7 & 29.7 & 46.7 & 9.3 & 6.67 & 6.08 \\
\hline $\mathbf{I}_{\mathbf{3}} \mathbf{M}_{\mathbf{3}}$ & 76.0 & 135.7 & 200.7 & 8.3 & 22.7 & 29.7 & 47.0 & 9.3 & 6.67 & 6.08 \\
\hline $\mathbf{I}_{3} \mathbf{M}_{4}$ & 75.7 & 139.7 & 211.0 & 8.7 & 23.0 & 31.0 & 48.0 & 9.3 & 7.00 & 6.47 \\
\hline $\mathbf{I}_{3} \mathbf{M}_{5}$ & 79.0 & 142.3 & 209.7 & 8.7 & 23.7 & 31.3 & 48.3 & 9.3 & 7.00 & 6.54 \\
\hline $\mathbf{I}_{3} \mathbf{M}_{6}$ & 80.0 & 146.0 & 214.7 & 9.3 & 24.7 & 32.0 & 48.7 & 9.7 & 7.33 & 6.83 \\
\hline $\mathbf{I}_{\mathbf{3}} \mathbf{M}_{\mathbf{7}}$ & 59.7 & 120.0 & 168.7 & 6.7 & 17.0 & 27.0 & 45.0 & 7.3 & 5.67 & 5.13 \\
\hline $\mathbf{I}_{\mathbf{3}} \mathbf{M}_{\mathbf{8}}$ & 66.0 & 131.3 & 186.0 & 7.3 & 19.0 & 29.7 & 45.7 & 8.3 & 6.33 & 5.74 \\
\hline S.Em \pm & 1.83 & 3.0 & 2.86 & 0.41 & 0.63 & 0.90 & 1.08 & 0.45 & 0.38 & 0.33 \\
\hline C.D. at $1 \%$ & NS & NS & NS & NS & NS & NS & NS & NS & NS & NS \\
\hline
\end{tabular}

I: Irrigation level - $\mathrm{I}_{1}$ : Once a day at 50 per cent of pan evaporation, $\mathrm{I}_{2}$ : Once a day at 75 per cent of pan evaporation,

$\mathrm{I}_{3}$ : Once a day at 100 per cent of pan evaporation, $\mathrm{M}$ : Media $-\mathrm{M}_{1}$ : Cocopeat, $\mathrm{M}_{2}$ : Perlite, $\mathrm{M}_{3}$ : Vermiculite, $\mathrm{M}_{4}$ :

Cocopeat + Perlite (50:50), $\mathrm{M}_{5}$ : Cocopeat + Vermiculite (50:50) $\mathrm{M}_{6}$ : Cocopeat + Perlite + Vermiculite (50:25:25),

$\mathrm{M}_{7}$ : Sole soil (Grown in earthen pot), $\mathbf{M}_{8}$ : Sole soil (Grown under normal condition) and NS: Non significant 
Int.J.Curr.Microbiol.App.Sci (2018) 7(4): 302-308

Table.2 Yield and economics as influenced by irrigation levels and different substrates

\begin{tabular}{|c|c|c|c|c|c|c|c|}
\hline Treatment & $\begin{array}{c}\text { Per cent } \\
\text { fruit set }(\%)\end{array}$ & $\begin{array}{c}\text { Fruit } \\
\text { weight (g) }\end{array}$ & $\begin{array}{l}\text { Fruit yield } \\
\left(t \mathbf{h a}^{-1}\right)\end{array}$ & $\begin{array}{l}\text { Per cent juice } \\
\text { recovery }\end{array}$ & $\begin{array}{c}\text { Gross returns } \\
\left(\text { Rs. } \text { ha }^{-1}\right)\end{array}$ & $\begin{array}{l}\text { Net returns } \\
\left(\text { Rs. }^{-1} \mathbf{a}^{-1}\right)\end{array}$ & B-C ratio \\
\hline \multicolumn{8}{|l|}{ Irrigation (I) } \\
\hline $\mathrm{I}_{1}$ & 88.3 & 43.4 & 64.7 & 55.63 & 776574 & 144566 & 1.24 \\
\hline$\overline{I_{2}}$ & 91.2 & 45.7 & 86.8 & 56.47 & 1041398 & 409390 & 1.65 \\
\hline $\mathbf{I}_{3}$ & 91.8 & 46.0 & 105.9 & 57.21 & 1270583 & 638576 & 2.03 \\
\hline S.Em \pm & 0.79 & 0.12 & 1.00 & 0.16 & 12057 & 12057 & 0.02 \\
\hline C.D. at $1 \%$ & 2.98 & 0.47 & 3.81 & 0.62 & 45735 & 45735 & 0.08 \\
\hline \multicolumn{8}{|l|}{ Media (M) } \\
\hline$M_{1}$ & 90.1 & 45.4 & 89.2 & 56.25 & 1070657 & 440213 & 1.70 \\
\hline$\overline{M_{2}}$ & 90.8 & 45.3 & 89.3 & 56.29 & 1071081 & 440636 & 1.70 \\
\hline $\mathbf{M}_{3}$ & 90.2 & 45.2 & 91.2 & 56.20 & 1094150 & 285235 & 1.35 \\
\hline $\mathbf{M}_{4}$ & 91.2 & 45.7 & 95.3 & 56.38 & 1144065 & 513621 & 1.81 \\
\hline $\overrightarrow{M_{5}}$ & 91.4 & 45.5 & 94.7 & 56.45 & 1136732 & 417053 & 1.58 \\
\hline $\mathbf{M}_{6}$ & 90.5 & 45.5 & 100.9 & 56.67 & 1210765 & 488308 & 1.68 \\
\hline $\mathbf{M}_{7}$ & 89.9 & 43.3 & 56.4 & 56.67 & 677343 & 220506 & 1.48 \\
\hline$\overline{\mathbf{M}_{8}}$ & 89.3 & 44.2 & 69.3 & 56.57 & 831351 & 374514 & 1.82 \\
\hline S.Em \pm & 1.28 & 0.20 & 1.64 & 0.27 & 19689 & 19689 & 0.03 \\
\hline C.D. at $1 \%$ & NS & 0.76 & 6.22 & NS & 74685 & 74685 & 0.13 \\
\hline \multicolumn{8}{|l|}{ Interaction } \\
\hline$I_{1} M_{1}$ & 87.94 & 43.80 & 66.3 & 55.59 & 795621 & 165176 & 1.26 \\
\hline $\mathbf{I}_{1} \mathbf{M}_{2}$ & 89.26 & 43.73 & 67.2 & 55.83 & 806145 & 175700 & 1.28 \\
\hline$I_{1} M_{3}$ & 89.21 & 43.40 & 69.3 & 55.06 & 831851 & 22937 & 1.03 \\
\hline$I_{1} M_{4}$ & 87.99 & 44.10 & 71.2 & 55.94 & 854120 & 223675 & 1.35 \\
\hline$I_{1} M_{5}$ & 89.27 & 43.67 & 69.8 & 55.69 & 837370 & 117691 & 1.16 \\
\hline$I_{1} M_{6}$ & 87.31 & 43.97 & 75.1 & 55.29 & 901386 & 178928 & 1.25 \\
\hline $\mathbf{I}_{1} \mathbf{M}_{7}$ & 88.25 & 41.40 & 48.4 & 55.85 & 580807 & 123971 & 1.27 \\
\hline $\mathbf{I}_{\mathbf{1}} \mathbf{M}_{8}$ & 87.33 & 42.87 & 50.4 & 55.75 & 605290 & 148454 & 1.32 \\
\hline $\mathbf{I}_{2} \mathbf{M}_{1}$ & 91.59 & 45.70 & 91.8 & 56.02 & 1101072 & 470628 & 1.75 \\
\hline $\mathbf{I}_{2} \mathbf{M}_{2}$ & 91.75 & 45.50 & 91.5 & 56.13 & 1097602 & 467158 & 1.74 \\
\hline $\mathrm{I}_{2} \mathrm{M}_{3}$ & 90.03 & 45.37 & 94.1 & 56.11 & 1129685 & 320771 & 1.40 \\
\hline $\mathbf{I}_{2} \mathbf{M}_{4}$ & 92.71 & 46.10 & 97.3 & 56.09 & 1167850 & 537406 & 1.85 \\
\hline $\mathbf{I}_{2} \mathbf{M}_{5}$ & 91.40 & 46.17 & 96.1 & 56.50 & 1152800 & 433121 & 1.60 \\
\hline $\mathrm{I}_{2} \mathrm{M}_{6}$ & 91.21 & 46.13 & 100.9 & 56.97 & 1210577 & 488119 & 1.68 \\
\hline $\mathrm{I}_{2} \mathrm{M}_{7}$ & 91.12 & 45.20 & 53.3 & 57.16 & 639198 & 182361 & 1.40 \\
\hline $\mathrm{I}_{2} \mathrm{M}_{8}$ & 90.00 & 45.50 & 69.4 & 56.82 & 832395 & 375559 & 1.82 \\
\hline $\mathbf{I}_{3} \mathbf{M}_{1}$ & 90.90 & 46.70 & 109.6 & 57.14 & 1315279 & 684834 & 2.09 \\
\hline $\mathbf{I}_{3} \mathbf{M}_{2}$ & 91.35 & 46.77 & 109.1 & 56.91 & 1309496 & 679051 & 2.08 \\
\hline $\mathbf{I}_{3} \mathbf{M}_{3}$ & 91.27 & 46.70 & 110.1 & 57.43 & 1320912 & 511998 & 1.63 \\
\hline $\mathbf{I}_{3} \mathbf{M}_{4}$ & 92.90 & 46.90 & 117.5 & 57.11 & 1410226 & 779781 & 2.24 \\
\hline $\mathrm{I}_{3} \mathrm{M}_{5}$ & 93.65 & 46.77 & 118.3 & 57.15 & 1420027 & 700348 & 1.97 \\
\hline $\mathbf{I}_{3} \mathbf{M}_{6}$ & 92.99 & 46.30 & 126.7 & 57.75 & 1520334 & 797877 & 2.10 \\
\hline $\mathbf{I}_{3} \mathbf{M}_{7}$ & 90.40 & 43.43 & 67.7 & 57.00 & 812023 & 355187 & 1.78 \\
\hline $\mathbf{I}_{3} \mathbf{M}_{8}$ & 90.52 & 44.33 & 88.0 & 57.15 & 1056366 & 599530 & 2.31 \\
\hline S.Em \pm & 2.22 & 0.35 & 2.84 & 0.81 & 34102 & 34102 & 0.06 \\
\hline C.D. at $1 \%$ & NS & 1.32 & 10.78 & NS & 129358 & 129358 & NS \\
\hline
\end{tabular}

I: Irrigation level - $\mathrm{I}_{1}$ : Once a day at 50 per cent of pan evaporation, $\mathrm{I}_{2}$ : Once a day at 75 per cent of pan evaporation,

$\mathrm{I}_{3}$ : Once a day at 100 per cent of pan evaporation, $\mathrm{M}$ : Media $-\mathrm{M}_{1}$ : Cocopeat, $\mathrm{M}_{2}$ : Perlite, $\mathrm{M}_{3}$ : Vermiculite, $\mathrm{M}_{4}$ :

Cocopeat + Perlite (50:50), $\mathrm{M}_{5}$ : Cocopeat + Vermiculite (50:50) $\mathrm{M}_{6}$ : Cocopeat + Perlite + Vermiculite (50:25:25),

$\mathrm{M}_{7}$ : Sole soil (Grown in earthen pot), $\mathrm{M}_{8}$ : Sole soil (Grown under normal condition) and NS: Non significant 
Similarly number of branches differed significantly in cocopeat + perlite + vermiculite at 50:25:25 (Table 1) as compared to others. Higher number of branches increased the number of leaves and improved light interception and photosynthetic rate.

However, the lower plant height $(151.4 \mathrm{~cm})$ and number of branches (24.0) were recorded (Table1) in sole soil grown in earthen pot due to higher bulk density, less water holding porosity and also less dissolved oxygen available in the root zone. Hence, the photosynthetic rate of the plant was declined which led to decreased yield of tomato.

\section{Per cent juice recovery}

Drip irrigation levels had a significant influence on per cent juice recovery of tomato fruits. Among irrigation levels, drip irrigation at 100 per cent PE recorded significantly higher per cent juice recovery of tomato fruits $(57.21 \%)$ as compared to 75 per cent PE (56.47\%) and 50 per cent PE (55.63\%). Similarly, substrate did not influence on per cent juice recovery of tomato. The interaction effects due to drip irrigation levels and substrates did not differ significantly among various treatment combinations. Similar findings were reported by Shinohara et al., (1999) and Borji and Jafarpour (2012).

\section{Interaction effect}

Interaction effect differed significantly for fruit weight and yield $\mathrm{ha}^{-1}$. The treatment combination of drip irrigation at 100 per cent $\mathrm{PE}$ with cocopeat + perlite + vermiculite at 50:25:25 $\left(\mathrm{I}_{3} \mathrm{M}_{6}\right)$ recorded significantly higher fruit weight $(46.77 \mathrm{~g})$ and fruit yield (126.7 $\mathrm{t}$ $\mathrm{ha}^{-1}$ ) as compared to other combinations. The higher yield might be due to the cumulative effect of higher number of fruits cluster ${ }^{-1}$, number of flower clusters plant ${ }^{-1}$ and number of flowers cluster ${ }^{-1}$. The increase in yield attributes was in turn due to better crop growth.

\section{Economics}

Drip irrigation at 100 per cent PE recorded (Table 2 significantly higher gross returns (Rs.1270583 ha ${ }^{-1}$ ), net returns (Rs. 638576 $\mathrm{ha}^{-1}$ ) and B-C ratio (2.03) as compared to others. The increase in gross returns, net returns and $\mathrm{B}-\mathrm{C}$ ratio were attributed to higher yields of tomato (Table 2). Similar findings were reported by Dunage et al., (2009).

Similarly, various substrates had a significant influence on economics of tomato. Cocopeat + perlite + vermiculite at 50:50:25 recorded significantly higher gross returns (Rs. $1210765 \mathrm{ha}^{-1}$ ) but it was on par with cocopeat + perlite at 50:50 (Rs. $1144065 \mathrm{ha}^{-1}$ ) and cocopeat + vermiculite at 50:50 (Rs. 1136732 $\left.\mathrm{ha}^{-1}\right)$. Mixed substrates with cocopeat + perlite at 50:50 recorded significantly higher net returns (Rs. $513621 \mathrm{ha}^{-1}$ ) as compared to others. Whereas, B-C ratio (1.82) was higher with conventionally grown tomato, but it was on par with cocopeat + perlite at 50:50 (1.81) perlite (1.70) and cocopeat (1.70). The B-C ratio in media grown tomato was less due to higher initial cost of substrates as compared to others. Similar results were obtained by Mazahreh et al., (2015) in cucumber.

The interaction effect had a significant influence on economics of tomato except B-C ratio. The combination of drip irrigation at 100 per cent PE with cocopeat + perlite + vermiculite at 50:25:25 $\left(\mathrm{I}_{3} \mathrm{M}_{6}\right)$ recorded higher gross returns (Rs. $1520334 \mathrm{ha}^{-1}$ ) and net returns (Rs.797877 ha ${ }^{-1}$ ) over other treatment combinations.

The results were concluded that drip irrigation at 100 per cent pan evaporation with cocopeat + perlite + vermiculite $(50: 25: 25)$ may be used in tomato to harvest maximum growth, 
yield and net returns. The next in order was cocopeat + perlite $(50: 50)$.

\section{References}

Borji, H and. Jafarpour, G. M., 2012, A comparison between tomato quality of mature-green and red- ripe stages in soilless culture. African J. Agric. Res., 7 (10): 1601-1603.

Butler and Oebker, 2006, Hydroponics as a hobby-growing plants without soil. Circular 844. Information Office, College of Agriculture, University of Illinois, Urbana, IL 6180.

Dunage, V. S. Balakrishnan, P. and Patil, M. G., 2009, Water use efficiency and economics of tomato using drip irrigation under net house conditions. Karnataka J. Agric. Sci., 22 (1): 133136.

Haddad, M., 2007, Effect of three substrates on growth, yield and quality of tomato by the use of geothermal water in the south of Tunisia. J. Food Agric. Environ., 5 (2): 175-178.

He, M., Chen, Z., Sakurai, K., Iwasaki, K., Shen, Y. and Zhou, J., 2003, Effect of differences in substrate formulations on cucumber growth under soilless bag culture in greenhouse. Soil Sci. Plant Nutr., 49 (5): 763-776.

Joseph, A. and Muthuchamy, I., 2014, Productivity, quality and economics of tomato (Lycopersicon esculentum mill.) cultivation in aggregate hydroponics $-\mathrm{a}$ case study from Coimbatore region of Tamilnadu. Indian J. Sci. Technol., 7(8): 1078-1086.

Mazahreh, N, Nejatian, A. and Mousa, M., 2015, Effect of different growing Media on Cucumber Production and Water Productivity in Soilless Culture under UAE Conditions. Merit Res. J. Agric. Sci. Soil Sci., 3 (9): 131-138.

Sardare and Admane, 2013. A review on plant without soil-hydroponics, Int. J. Res. Engg. Tech., 2 (3): 299-304.

Sezen, S. M., Celikel, G., Yazar, A., Tekinand, S. and Kapur, S., 2010, Effect of irrigation management on yield and quality of tomatoes grown in different soilless media in a glasshouse. Sci. Res. and Essay 5 (1): 41-48.

Shinohara, Y. Hata, T., Maruo, T. and Hohjo, M., Ito, T., and Papadopoulos, A. P. 1999, Chemical and physical properties of the coconut fiber substrates and the growth and productivity of tomato (Lycopersicon esculentum Mill) plants. Acta Hort. 481: 145- 149.

\section{How to cite this article:}

Parameshwarareddy, R., S.S. Angadi, R.H. Patil and Biradar, M.S. 2018. Influence of Drip Irrigation Levels and Soilless Media on the Growth, Productivity and Economics of Greenhouse Grown Tomato. Int.J.Curr.Microbiol.App.Sci. 7(04): 302-308. doi: https://doi.org/10.20546/ijcmas.2018.704.034 